\title{
Study protocol: a pilot quasi-experimental trial of tele-rehabilitation and tele-drain care post-mastectomy
}

\author{
Miho Asano ${ }^{1 *}$, Gerald Choon-Huat Koh ${ }^{1}$, Preetha Madhukumar ${ }^{2,3,4}$, Gladys Yu Hui Teng ${ }^{5}$, Petrina Li Ling Liew ${ }^{5}$,
} Saraswathi Nagalingam ${ }^{6}$, May Leng Mabel Tan ${ }^{7}$, Yee Sien $\mathrm{Ng}^{8,9}$ and Benita Kiat Tee $\operatorname{Tan}^{2,3,4}$

\begin{abstract}
Background: Breast cancer is the leading cancer affecting women in Singapore. Its survivors commonly experience decline in physical function and quality of life post-mastectomy, due to their upper limb morbidity and wound issues. Rehabilitation can address the aforementioned issues. When rehabilitation is accessible and easy to adhere, it can optimize recovery.

Home-based tele-rehabilitation guided by healthcare professionals and self-managed by patients can potentially optimize the patients' adherence to rehabilitation and recovery. With that in mind, a team of breast cancer specialists (oncologists, nurses, and therapists) in Singapore has developed one of the first tele-rehabilitation systems for local women undergoing a unilateral mastectomy. To our knowledge, no such systems have been evaluated or proven effective as a treatment option among local breast cancer patients with acute disabilities.
\end{abstract}

Methods: This is a pilot quasi-experimental trial that aims to evaluate the feasibility of tele-rehabilitation and teledrain care compared to usual care. Up to 40 patients (20 per group) will be recruited for this trial. They will be assigned to an intervention group that receives rehabilitation via a tele-rehabilitation system or a control group that receives rehabilitation in person at their clinic. The primary outcome of this trial is rehabilitation participation (i.e., the time spent on rehabilitation). The secondary outcomes are upper extremity functioning, perceived health, and quality of life.

Discussion: As part of this pilot trial, patients who opt in for the tele-rehabilitation will be asked to share their experience with and thoughts on the tele-rehabilitation system. With the evidence obtained from the telerehabilitation patients of this trial, we will be able to improve the current system for our future trial. Further, our additional data on rehabilitation participation, physical function, and quality of life will help us design a sufficiently powered future main trial.

Trial registration: The trial was approved by the National Healthcare Group's Domain Specific Review Board (\#2019/00283) and registered with www.ClinicalTrials.gov (\#NCT04269967) in February 2020.

Keywords: Tele-rehabilitation, Pilot trial, Breast cancer

\footnotetext{
* Correspondence: epham@nus.edu.sg

'Saw Swee Hock School of Public Health, National University of Singapore,

12 Science Drive 2, \#09-01, Singapore 117549, Singapore

Full list of author information is available at the end of the article
}

C C The Author(s). 2021 Open Access This article is licensed under a Creative Commons Attribution 4.0 International License, which permits use, sharing, adaptation, distribution and reproduction in any medium or format, as long as you give appropriate credit to the original author(s) and the source, provide a link to the Creative Commons licence, and indicate if changes were made. The images or other third party material in this article are included in the article's Creative Commons licence, unless indicated otherwise in a credit line to the material. If material is not included in the article's Creative Commons licence and your intended use is not permitted by statutory regulation or exceeds the permitted use, you will need to obtain permission directly from the copyright holder. To view a copy of this licence, visit http://creativecommons.org/licenses/by/4.0/ The Creative Commons Public Domain Dedication waiver (http://creativecommons.org/publicdomain/zero/1.0/) applies to the data made available in this article, unless otherwise stated in a credit line to the data. 


\section{Background}

Breast cancer is the leading cancer affecting women in Singapore. It accounts for approximately 30\% of cancer diagnosis among women in the nation [1]. The majority of breast cancer diagnoses in Singapore are at stage 1 and 2 with a high 5 -year survival rate [1]. Treatment recommendations for breast cancer depend on various factors (e.g., stage of the cancer, size of the tumor, and personal preferences). In early stage of breast cancer, surgical procedure (such as lumpectomy or mastectomy) is often the first treatment option to be recommended. Further, women may elect to undergo mastectomy for different reasons (such as their hesitance toward radiation therapy and fear of recurrence) [2, 3]. Rates of mastectomy ranged from 43 to $59 \%$ locally between 2001 and 2010 [4]. Most breast cancer survivors experience decline in physical function and quality of life (QoL) due to their upper limb morbidity and wound issues after mastectomy [5].

Rehabilitation (e.g., physical and occupational therapy) is known to maintain or improve physical function and QoL [6]. It can address common issues (e.g., lymphedema, decreased endurance, and joint stiffness) that breast cancer survivors may experience after their surgery. For example, individuals with primary carcinoma who participated in post-surgery rehabilitation in a nonrandomized controlled trial showed a significant improvement in various functional outcomes as compared to those who did not participate in any rehabilitation [7]. The outcomes included physical, cognitive, and social functions [7]. The current post-surgery care for breast cancer patients in Singapore includes arm exercises, arm and hand care, and self-arm massage [8].

When rehabilitation (services) is accessible and easy to adhere, it can optimize patients' recovery post-surgery. However, a Singaporean mixed methods study of 70 patients discharged from inpatient rehabilitation found their common intention to discontinue their rehabilitation after their hospital discharge due to foreseeable barriers (e.g., accessibility, cost, and inconvenience), while the majority acknowledged the benefits of rehabilitation [9].

Telemedicine (or telehealth) refers to the use of telecommunications and technology to deliver health services (e.g., rehabilitation) outside of traditional settings (e.g., patients' home) [10]. Tele-rehabilitation (a homebased telehealth service) guided by healthcare professionals and self-managed by patients can potentially provide a solution to the aforementioned barriers, which may lead to improved participation in rehabilitation after hospital discharge [11]. A few telemedicine studies reported that individuals who were diagnosed with breast cancer and received their rehabilitation using telecommunications and technology showed an equal or a higher amount of improvement in their health status compared to those who received their rehabilitation in a traditional way [12-14].

\section{Aims}

The first aim of this study is to gather preliminary evidence for the feasibility of a tele-rehabilitation and teledrain care among women who undergo a unilateral mastectomy for breast cancer. The second aim is to understand the lived experience of breast cancer diagnosis, treatment, and recovery process.

\section{Methods \\ Study design}

This is a pilot quasi-experimental trial. Patients will be placed into the intervention group (tele-rehabilitation) or control group (usual rehabilitation care) based on their preference. The total length of the study period will be 15 weeks; patients will undergo a 3-week drain care period (post-surgery) which will be followed by a 12week rehabilitation period. A baseline assessment will be conducted prior to commencing rehabilitation (approximately 3 weeks from the surgery) and a follow-up (exit) assessment will be conducted at the end of their rehabilitation period (approximately 12 weeks from the baseline assessment). An additional qualitative study is embedded within the trial to gain a deeper understanding of the lived experience of women who are diagnosed with breast cancer to achieve the second aim.

\section{Patients (participants)}

We plan to recruit up to 40 patients (20 per group) from the Singapore General Hospital (SGH) and National Cancer Centre Singapore (NCCS), where they will be receiving preoperative, surgical, postoperative, and rehabilitation care. Patients are recruited from multiple clinics, under a single healthcare system in Singapore, consisting of a multi-ethnic Asian population.

Patients are eligible to participate in the trial when they meet the following four criteria: (i) female 21 years of age and older, (ii) undergoing or underwent a recent unilateral breast surgery (wide excision or simple mastectomy) with lymph nodes removed, (iii) speak and write English or Mandarin, and (iv) no past or active psychiatric condition as assessed by a referring clinician. Patients are ineligible to participate if they meet one of the following four criteria: (i) having breast reconstruction, (ii) having legal blindness or severe visual impairment, (iii) having life expectancy of less than 3 months, or (iv) having previous upper limb injury or conditions that limit upper limb range of motion (shoulder flexion (< $\left.150^{\circ}\right)$ or elbow extension/flexion $\left(<0 / 145^{\circ}\right)$ respectively). 
Procedure (recruitment, allocation, and data collection)

Potential participants will be approached by a breast care nurse and/or doctor (who will be providing the patient care related to breast cancer and mastectomy) during their pre-operation counseling session. These nurses and doctors will provide their patients with our study invitation letter. The patients will be asked to contact the research team directly using the contact information provided in the study invitation letter if they are interested in the study. Alternatively, if the patients agree, the nurses and doctors will obtain patients' contact information (i.e., first or last name with an email address or a phone number only) which will be forwarded to the research team to initiate contact.

If patients agree to participate in the trial, they will complete the informed consent process with a trained member of the research team. The informed consent process can occur anytime between the time of their surgical consultation and 5 days post-surgery. After the informed consent process is completed, patients will self-assign themselves to the intervention group (tele-rehabilitation) or the control group (usual rehabilitation). The prescription and administration of drain care (education) and rehabilitation for both groups of patients are determined and managed by an assigned nurse and therapist per usual practice.

All consented patients will be asked to complete two assessments with a trained member of the research team at their home (or at the university where the research is being conducted). The first (baseline) assessment will take place approximately 3 -week post-surgery after completing drain-care (education) period (i.e., prerehabilitation assessment). The second (exit) assessment will take place after completing a 12-week physical rehabilitation period (i.e., post-rehabilitation assessment) (refer to Fig. 1 for an overview of the study outlining the timing of baseline and exit assessments). No patients, care providers, assessors, or analysts will be blinded. Any adverse events, although unanticipated, will be discussed within the team and reported to the National Healthcare Group Domain Specific Review Board within seven calendar days.

Tele-rehabilitation (intervention) and usual care (control) Patients in the intervention group will have the telerehabilitation system for up to 15 weeks. The first 3 weeks will be for drain care video education (post-mastectomy) and the following 12 weeks will be for physical tele-rehabilitation (including shoulder and arm exercise, and self-arm massage). During the physical rehabilitation period, patients (in the tele-rehabilitation group) and/or an assigned occupational therapist may arrange up to five 30-min video conferences (the number of sessions will be determined by an assigned therapist, as per request or per need of each patient).

Patients in the control group will receive the same instructions (for drain care education as well as shoulder and arm exercise and self-arm massage) by their multidisciplinary rehabilitation team but without remote support with the tele-rehabilitation and tele-drain care system. Their drain care education and physical rehabilitation sessions will instead take place in-person at their clinic with their assigned nurse and therapist (refer to Table 1 for brief descriptions of drain care education with a nurse and rehabilitation with an occupational therapist).

\section{Measurements}

A pilot study is conducted to test a set of feasibility objectives to warrant the viability of the main trial [15]. Based on an expected attrition or non-response rate, it is common to inflate the trial sample size for $10-20 \%$ [16]. The primary outcome of our pilot feasibility study is patients' participation in the study and prescribed rehabilitation (i.e., the time spent on rehabilitation). It is a process assessment where both groups of patients will be asked to keep records of their participation in rehabilitation using a diary (logbook). The data on rehabilitation (e.g., the number of the rehabilitation sessions that they have and the duration of each session) will be collected once at the post-rehabilitation assessment (i.e., the study participation rate). Further, our tele-rehabilitation system will track the frequency and duration of the system use for the intervention group. The attrition rate of $20 \%$ or less (or the completion rate of $80 \%$ or above) is considered the criterion for the success of feasibility for our study.

In addition, all patients will be asked to complete a semi-structured qualitative interview about their experience with the recent diagnosis, rehabilitation, and recovery process at the end of the trial (after the 12-week rehabilitation period). For the additional qualitative interview, patients will be asked a series of simple openended questions which may include the following: (1) tell us about how you navigated your diagnosis, (2) how do you describe rehabilitation that you participated in over the past 12 weeks, and (3) how has your recovery process been-as expected or unexpected? The interview data will be digitally recorded with the patient's permission and transcribed for qualitative analysis.

The secondary outcomes of this trial are upper extremity functioning, perceived health, and QoL. Upper extremity functioning will be measured by shoulder active range of motion (AROM) [17], arm circumference (AC) [18], the Quick Disabilities of Arm, Shoulder \& Hand (QuickDASH) [19]. Perceived health will be measured by the EuroQoL-5D (EQ-5D-3L) [20] and QoL 


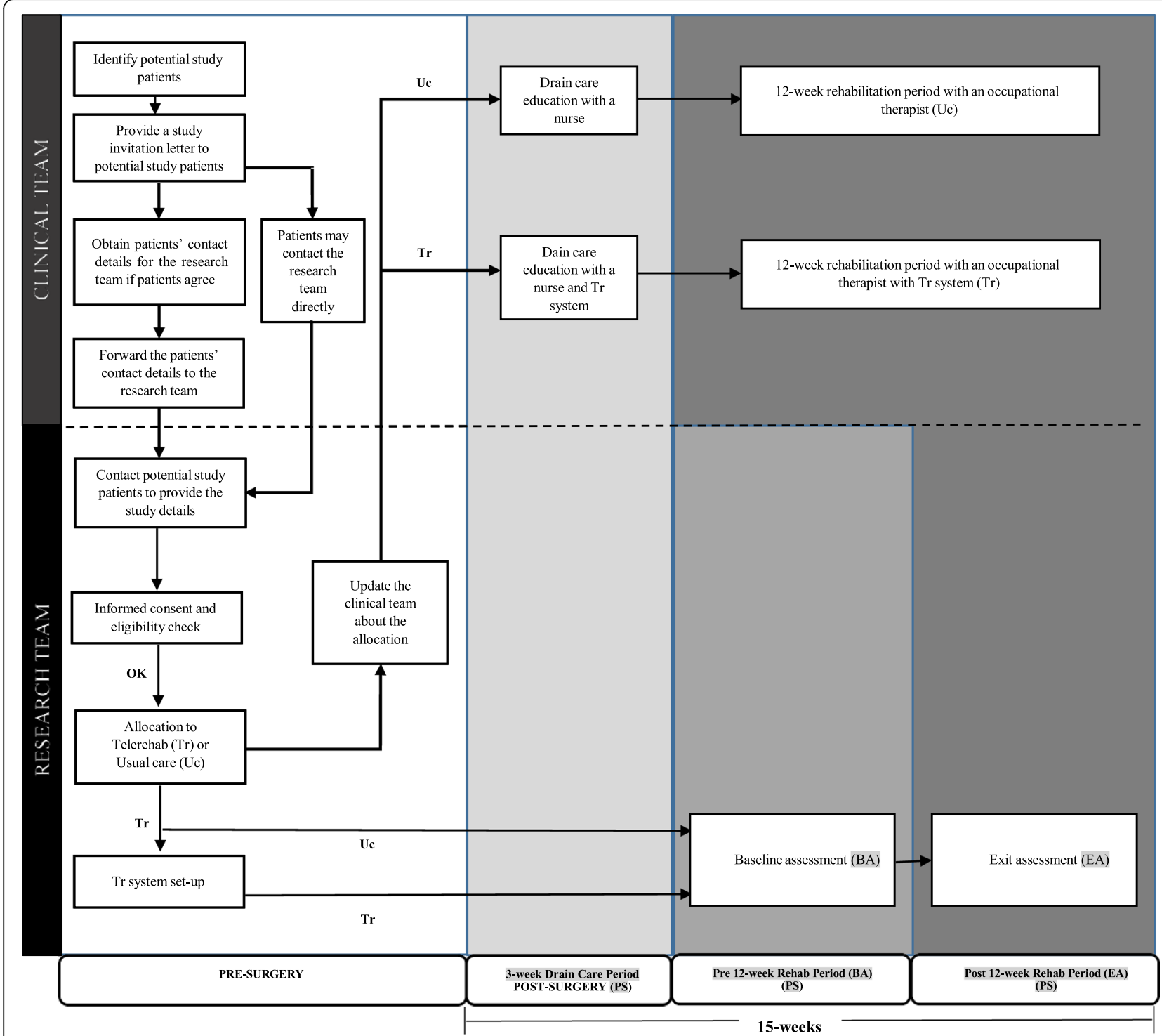

Fig. 1 An overview of the study

will be measured by the European Organization for Research and Treatment of Cancer Quality of Life Questionnaire Core 30 (EORTC QLQ-C30) [21]. For the secondary outcomes, all patients will be asked to complete all five measures with a trained member of the research team at both pre- and post-rehabilitation assessment. We hypothesize that both groups will show similar improvements across all secondary outcome measures.

\section{Active range of motion}

Patients will have their AROM (pain-free) measured for shoulder flexion, extension, and abduction on both arms using goniometry [17]. AROM for each action will be measured twice. The measurements per action per arm will be averaged for data analysis.

\section{Mid upper arm circumference measurement}

The MUAC will be specifically noted by measuring the arm circumference at the mid-point between the tip of the shoulder and tip of the elbow (acromion and olecranon process) [18]. Each measure will be taken twice on both arms by a trained member of the research team. The measurement per arm will be averaged for analysis.

\section{Self-reported upper extremity disability}

The QuickDASH is an 11-item questionnaire designed to assess self-reported disability of the arm, shoulder, and hand among individuals with upper extremity disorders [19]. Its total score may range from 0 (no disability) to 100 (most severe disability) [22]. The QuickDASH is reported to have good internal consistency and testretest reliability $[19,23]$. 
Table 1 Brief descriptions of drain care education with a nurse and rehabilitation with an occupational therapist

\begin{tabular}{|c|c|c|}
\hline Drain Care Education & Arm and Shoulder Massage & Exercise \\
\hline $\begin{array}{l}\text { Drain care education provides information on } \\
\text { how to manage the drainage devices and fluids } \\
\text { collected post-mastectomy. These include } \\
\text { proper and safe physical handling of the drain- } \\
\text { age devices, and proper sequences to empty, } \\
\text { measure, and dispose fluids from the drainage } \\
\text { devices. }\end{array}$ & $\begin{array}{l}\text { Arm and shoulder massage are performed with } \\
\text { the aim of managing edema (reducing arm } \\
\text { swelling) post-mastectomy. They consist of a } \\
\text { series of actions to facilitate pushing fluid out of } \\
\text { the affected areas back into the bloodstream, } \\
\text { therefore preventing fluid accumulation and } \\
\text { limb swelling. }\end{array}$ & $\begin{array}{l}\text { The exercises are performed with the aim of } \\
\text { maintaining the active range of motion of the } \\
\text { upper limbs, which concurrently helps to drain } \\
\text { fluid. The exercises involve deep breathing and } \\
\text { a series of movements including shoulder } \\
\text { elevation, retraction, protraction and flexion, and } \\
\text { elbow flexion and extension. }\end{array}$ \\
\hline \multicolumn{3}{|l|}{ Sample Instructions } \\
\hline $\begin{array}{l}\text { Step 1. Wash your hand } \\
\text { Step 2. Prepare your requisites } \\
\text { Step 3. Drain fluid for measurement } \\
\text { Step 4. Measure and record the amount of } \\
\text { fluid drained, before disposal (twice a day) }\end{array}$ & $\begin{array}{l}\text { Step 1. Take a deep breath } \\
\text { Step 2. Place your affected arm on your } \\
\text { shoulder with your elbow pointing to your side } \\
\text { Step 3. Using the other hand, place it below } \\
\text { your armpit } \\
\text { Step 4. Gently sweep downwards past your } \\
\text { waistline } \\
\text { Step 5. Please ensure your palm has good } \\
\text { contact with your body as you sweep } \\
\text { downwards } \\
\text { Step 6. Repeat this } 20 \text { times } \\
\text { Note. The arm/hand doing the massage is the } \\
\text { contralateral side massaging the affected side. }\end{array}$ & $\begin{array}{l}\text { Step 1. Begin the exercise by clasping both } \\
\text { hands and bringing them to the chest } \\
\text { Step 2. Slowly bring both arms forward, making } \\
\text { sure that your elbows are straight } \\
\text { Step 3. Repeat this } 5 \text { times }\end{array}$ \\
\hline
\end{tabular}

\section{Perceived health}

The EQ-5D-3L is a questionnaire designed to measure self-perceived health status of individuals [20]. It consists of five dimensions (i.e., mobility, self-care, usual activities, pain/discomfort, and anxiety/depression). Individuals are asked to select one of three responses for each domain (no problems, some problems, extreme problems/unable to perform) to describe their health status. The five responses are summed and converted to a health utility index value which ranges from -0.171 to 1 . Higher values indicate better health status. The EQ-5D-3L has been shown to have good test-retest reliability [24].

\section{QoL}

The EORTC QLQ-C30 is a QoL measure specifically designed for cancer patients. It consists of 30 items which form functional scales, a global health status/QoL scale, and symptoms scales (including financial difficulty) [21]. Scores of all scales and single-item measures range from 0 to 100 [25]. For the functioning scales and global QoL scales, higher scores indicate better functioning; for the symptom scales, higher scores indicate higher symptom burden. The EORTC QLQ is reported to have acceptable test-retest reliability and good construct validity with the 36-Item Short Form Survey [26, 27].

\section{Analysis}

For quantitative data, we will report means and standard deviations for parametric data, medians and interquartile ranges for non-parametric data, and numbers and percentages of all categorical variables for descriptive purposes. Further, inferential statistics (e.g., $T$ test, chi-square test) will be performed to compare the outcome measures between the two groups. We will perform a random data (entry) check by the secondary data management personnel (who did not collect the data or complete the initial data entry). Missing data will not be replaced or imputed.

For the qualitative data, we will conduct inductive analysis that involves discovering patterns, themes, and categories related to the lived experience of breast cancer diagnosis, rehabilitation, and recovery process (using five steps of qualitative data analysis outlined by Miles and Huberman) [28].

\section{A power calculation}

The data obtained from this pilot trial will be used to estimate a sufficient sample size for our future main trial. The existing literature suggests the minimum sample size of 10 to 15 participants per group [29] or $10 \%$ of a main trial's sample size [30] to be sufficient for a pilot feasibility study. Considering such evidence, we plan to recruit a minimum of 10 and a maximum of 20 patients per group for this pilot trial.

\section{Discussion}

Our pilot trial is designed to evaluate the feasibility of one of the first tele-rehabilitation systems for women undergoing a unilateral mastectomy developed in Singapore by a multidisciplinary team (e.g., surgery, oncology, rehabilitation, nursing, and engineering). To our knowledge, there is no published trial that assessed and proved the effectiveness of such systems as a treatment option among breast cancer patients with acute disabilities.

As part of this pilot trial, patients who opt in for the tele-rehabilitation will be asked to share their experience with and thoughts on the tele-rehabilitation system. 
With the evidence obtained from the tele-rehabilitation patients of this trial, we will be able to improve the current system for our future trial. Further, our additional data on rehabilitation participation, physical function, and quality of life will help us design a sufficiently powered future main trial. In the long term, if successful, we could have a safe and effective tele-rehabilitation system to implement as part of our regular healthcare services and optimize patients' recovery in the comfort and privacy of their home as well as at their own convenience.

\section{Trial status}

The trial obtained the institutional review board approval (\#2019/00283) and registered with www. ClinicalTrials.gov (\#NCT04269967) in February 2020. The study recruitment began in November 2020 and data collection is currently ongoing.

\section{Abbreviations}

AC: Arm circumference; AROM: Active range of motion; DASH: Disabilities of Arm, Shoulder, and Hand; EORTC QLQ-C30: European Organization for Research and Treatment of Cancer Quality of Life Questionnaire Core 30; EQ5D-3 L: EuroQoL-5D-3L; MUAC: Mid upper arm circumference measurement QoL: Quality of life; ROM: Range of motion

\section{Acknowledgements}

The authors would like to thank their research team, Mr. Yogaprakash Kumar (an engineer), Ms. Min Geng Ng and Mr. Nicklaus Koh (research assistants), and Mr. Nizar Mohamad and Ms. Joanna Yeo (research associates) for their assistance on the protocol preparation for publication and upcoming collaborative work

\section{Authors' contributions}

All authors contributed to the development of this trial. The first and corresponding author drafted this protocol for all co-authors and senior author to review, revise, and approve for publication. The author(s) read and approved the final manuscript.

\section{Funding}

The trial is supported jointly by the Singapore Cancer Society and the Ministry of Education Academic Research Fund Tier 1 (PI. Miho Asano). These sponsors have no roles (influences) on our study design; data collection, management, analysis, and interpretation; or manuscript development and publication.

\section{Availability of data and materials}

This is a protocol of a pilot trial (version 1) which is in progress; therefore, no data are currently available.

\section{Ethics approval and consent to participate}

The protocol for this trial was reviewed and approved by the National Healthcare Group Domain Specific Review Board (\#2019/00283). Written informed consent will be obtained from every patient who participates in this trial by a trained member of the research team.

\section{Consent for publication}

No individual details, images, or videos will be published as part of this trial.

\section{Competing interests}

No authors have competing interests to declare.

\section{Author details}

'Saw Swee Hock School of Public Health, National University of Singapore, 12 Science Drive 2, \#09-01, Singapore 117549, Singapore. ${ }^{2}$ Duke-NUS Breast Centre, 20 College Rd, Singapore 169856, Singapore. ${ }^{3}$ Department of Breast
Surgery, Singapore General Hospital, 10 Hospital Blvd, Singapore 168582, Singapore. ${ }^{4}$ Department of Breast Surgery, National Cancer Centre Singapore, 11 Hospital Crescent, Singapore 169610, Singapore. ${ }^{5}$ Department of Occupational Therapy, Singapore General Hospital, 10 Hospital Blvd, Singapore 168582, Singapore. ${ }^{6}$ Nursing Division Singapore General Hospital, 10 Hospital Blvd, Singapore 168582, Singapore. 'Department of Nursing, National Cancer Centre Singapore, 11 Hospital Crescent, Singapore 169610, Singapore. ${ }^{8}$ Duke-Nus Medical School, National University of Singapore, 8 College Road, Singapore 169867, Singapore. ${ }^{9}$ Department of Rehabilitation Medicine Singapore General Hospital, 10 Hospital Blvd, 168582 Singapore, Singapore.

Received: 9 July 2020 Accepted: 18 January 2021 Published online: 01 February 2021

\section{References}

1. National Registry of Diseases Office. Singapore Cancer Registry annual registry report 2015. Singapore: Health Promotion Board; 2017. p. 43.

2. McGuire KP, Santillan AA, Kaur P, Meade T, Parbhoo J, Mathias M, et al. Are mastectomies on the rise? A 13-year trend analysis of the selection of mastectomy versus breast conservation therapy in 5865 patients. Ann Surg Oncol. 2009;16:2682-90. https://doi.org/10.1245/s10434-009-0635-x.

3. Miller KD, Siegel RL, Lin CC, Mariotto AB, Kramer JL, Rowland JH, et al. Cancer treatment and survivorship statistics, 2016. CA Cancer J Clin. 2016:66: 271-89. https://doi.org/10.3322/caac.21349.

4. Chan PM, Choo BA, Zhang T, Seah MD, Chen JJ, Lu SQ, et al. Mastectomy rates remain high in Singapore and are not associated with poorer survival after adjusting for age. SpringerPlus. 2015;4(1):685.

5. Hidding JT, Beurskens $\mathrm{CH}$, van der Wees PJ, van Laarhoven HW, Nijhuis-van der Sanden MW. Treatment related impairments in arm and shoulder in patients with breast cancer: a systematic review. PLoS One. 2014;9:e96748.

6. Spence RR, Heesch KC, Brown WJ. Exercise and cancer rehabilitation: a systematic review. Cancer Treat Rev. 2010;36:185-94. https://doi.org/10. 1016/j.ctrv.2009.11.003

7. Leclerc A-F, Foidart-Dessalle M, Tomasella M, Coucke P, Devos M, Bruyere O, et al. Multidisciplinary rehabilitation program after breast cancer: benefits on physical function, anthropometry and quality of life. Eur J Phys Rehabil Med. 2017;53(5):633-42

8. SingHealth Duke-NUS Breast Centre. (2019). Breast cancer - diagnosis to treatment - post-surgery care. https://www.singhealth.com.sg/patient-care/ conditions-treatments/breast-cancer-diagnosis-treatment/post-surgery-care

9. Chen AW, Koh YT, Leong SW, Ng LW, Lee PS, Koh GC. Post community hospital discharge rehabilitation attendance: self-perceived barriers and participation over time. Ann Acad Med Singapore. 2014;43:136-44.

10. World Health Organization. Telemedicine: opportunities and developments in member states: report on the second global survey on ehealth 2009. Geneva: World Health Organization; 2010.

11. Koh GC, Yen SC, Tay A, Ng YS, Caves K, Tai BC, et al. Comparing the effectiveness of tele-rehabilitation and center-based rehabilitation: STARS clinical trial. Arch Phys Med Rehabil. 2018;99:e44. https://doi.org/10.1016/j. apmr.2018.07.152.

12. Borosund E, Cvancarova M, Moore SM, Ekstedt M, Ruland CM. Comparing effects in regular practice of e-communication and web-based selfmanagement support among breast cancer patients: preliminary results from a randomized controlled trial. J Med Internet Res. 2014;16:e295. https://doi.org/10.2196/jmir.3348.

13. Galiano-Castillo N, Cantarero-Villanueva I, Fernandez-Lao C, Ariza-Garcia A, Diaz-Rodriguez L, Del-Moral-Avila $\mathrm{R}$, et al. Telehealth system: a randomized controlled trial evaluating the impact of an internet-based exercise intervention on quality of life, pain, muscle strength, and fatigue in breast cancer survivors. Cancer. 2016;122:3166-74. https://doi.org/10.1002/cncr. 30172.

14. Uhm KE, Yoo JS, Chung SH, Lee JD, Lee I, Kim Jl, et al. Effects of exercise intervention in breast cancer patients: is mobile health ( $\mathrm{mHealth}$ ) with pedometer more effective than conventional program using brochure? Breast Cancer Res Treat. 2017;161:443-52. https://doi.org/10.1007/s10549016-4065-8

15. Thabane $L$, Lancaster $G$. A guide to the reporting of protocols of pilot and feasibility trials; 2019. p. 37

16. Machin D, Campbell MJ, Walters SJ. Medical statistics; 2007. 
17. Gajdosik RL, Bohannon RW. Clinical measurement of range of motion: review of goniometry emphasizing reliability and validity. Phys Ther. 1987; 67(12):1867-72.

18. Armer JM, Stewart BR. A comparison of four diagnostic criteria for lymphedema in post-breast cancer population. Lymphat Res Biol. 2005;2(4): 208-17.

19. Beaton DE, Wright JG, Katz JN, Upper Extremity Collaborative Group. Development of the QuickDASH: comparison of three item-reduction approaches. J Bone Joint Surg Am. 2005;87(5):1038-46. https://doi.org/10. 2106/JBJS.D.02060

20. EuroQol Research Foundation. EQ-5D-3L user guide (Internet) (Version 6.0). Rotterdam: EuroQol Research Foundation; 2018. p. 34. Available from https://euroqol.org/publications/user-guides

21. Aaronson NK, Ahmedzai S, Bergman B, Bullinger M, Cull A, Duez NJ, et al. The European Organisation for Research and Treatment of Cancer QLQ-C30: a quality-of-life instrument for use in international clinical trials in oncology. J Natl Cancer Inst. 1993:85(5):365-76.

22. Gummesson C, Ward MM, Atroshi I. The shortened disabilities of the arm, shoulder and hand questionnaire (QuickDASH): validity and reliability based on responses within the full-length DASH. BMC Musculoskelet Disord. 2006; 7:44. https://doi.org/10.1186/1471-2474-7-44.

23. LeBlanc M, Stineman M, DeMichele A, Stricker C, Mao JJ. Validation of QuickDASH outcome measure in breast cancer survivors for upper extremity disability. Arch Phys Med Rehabil. 2014;95:493-8. https://doi.org/ 10.1016/j.apmr.2013.09.016.

24. Kim SH, Jo MW, Lee JW, Lee HJ, Kim JK. Validity and reliability of EQ-5D-3L for breast cancer patients in Korea. Health Qual Life Outcomes. 2015;13:203. https://doi.org/10.1186/s12955-015-0399-x.

25. Fayers PM, Aaronson NK, Bjordal K, Groenvold M, Curran D, Bottomley A, on behalf of the EORTC Quality of Life Group. The EORTC QLQ-C30 scoring manual. 3rd ed. Brussels: European Organisation for Research and Treatment of Cancer; 2001.

26. Luo N, Fones CS, Lim SE, Xie F, Thumboo J, Li SC. The European Organization for Research and Treatment of Cancer Quality of Life Questionnaire (EORTC QLQ-c30): validation of English version in Singapore Qual Life Res. 2005;14:1181-6. https://doi.org/10.1007/s11136-004-4782-z.

27. Michels FAS, Latorre Mdo R, Maciel MS. Validity, reliability and understanding of the EORTC-C30 and EORTC-BR23, quality of life questionnaires specific for breast cancer. Rev Bras Epidemiol. 2013;16:35263. https://doi.org/10.1590/S1415-790X2013000200011.

28. Miles MB, Huberman AM, editors. An expanded sourcebook: qualitative data analysis. 2nd ed. Thousand Oaks: Sage Publications; 1994.

29. Hertzog MA. Considerations in determining sample size for pilot studies. Res Nurs Health. 2008;31:180-91. https://doi.org/10.1002/nur.20247.

30. Lackey NR, Wingate AL. The pilot study: one key to research success. In: Brink PJ, Wood MJ, editors. Advanced design in nursing research. 2nd ed. Thousand Oaks: Sage; 1998. p. 375-86.

\section{Publisher's Note}

Springer Nature remains neutral with regard to jurisdictional claims in published maps and institutional affiliations.

Ready to submit your research? Choose BMC and benefit from:

- fast, convenient online submission

- thorough peer review by experienced researchers in your field

- rapid publication on acceptance

- support for research data, including large and complex data types

- gold Open Access which fosters wider collaboration and increased citations

- maximum visibility for your research: over $100 \mathrm{M}$ website views per year

At $\mathrm{BMC}$, research is always in progress.

Learn more biomedcentral.com/submissions 